\title{
Manganous-zinc ferrites synthesized out of $\mathrm{MnO}^{\dagger \dagger}$
}

\author{
C E DESHPANDE, P P BAKARE and M N SANKARSHANA \\ MURTHY*i \\ Physical Chemistry Division. National Chemucal Laboratory, Poona $\$ 11008$, India \\ 1 Materials Research Laboratory, Indian Institute of Science, Bangalore 560012 , India. \\ MS received 6 July 1982; revised 24 September 1982
}

\begin{abstract}
Manganous-zinc ferrites are best prepared directly from $\mathrm{MnO}$ and can be conveniently pressed with non-volatilizable binders such as polyvinyl alcohol which however can be burnt out without oxidizing the $\mathrm{Mn}^{2+}$ in the ferrite by sintering in a static atmosphere generated by the thermal dissociation of active ferric oxide pellets placed out of contact with the ferrite in the closed sintering unit. Such a process eliminates racks and rejections in the sintered pieces besides giving very satisfactory and reproducible results.
\end{abstract}

Keywords. Manganous-zinc ferrites; sintering; dimination of cracks.

\section{Introduction}

In two previous communications (Murthy at al 1978, 1979 which will be referred to hereafter as papers 1 and 2 respectively) the preparation of manganous-zinc ferrites directly from $\mathrm{MnO}$ itself has been dealt with. Paper 1 deals with the use of $\mathrm{MnO}$ protected from oxidation in a closed system, while paper 2 describes the use of $\mathrm{MnO}$ stabilized by solid solution with $\mathrm{ZnO}$. In either case the bivalency of manganese is maintained right from the start. It is worth comparing manganous-zinc ferrites prepared in this way with those prepared out of a stable higher oxide of manganese. In the latter method which is quite' popular the higher valency of manganese is reduced to two at the time of sintering (Strivens and Chol 1971; Natansohn and Baird 1969). It would be interesting to see whether this is the same as retaining manganese in the correct valency state right from the beginning of the preparative process.

Even if it is a preferred practice to prepare manganous-zinc ferrites without allowing the manganese to be in any valency state higher than the bivalent at any stage, there still arises the problem of burning the binders employed in pressing the ferrite powders: the $\mathrm{Mn}^{2+}$ may get oxidized in the process. Papers 1 and 2 dealt with this problem in two ways. One was to use a volatilizable binder capable of being distilled off in an inert atmosphere; alternatively the ferrite was allowed to get oxidized while burning a nonvolatilizable binder such as PVA, and subsequently the $\mathrm{Mn}^{3+}$ was reduced to $\mathrm{Mn}^{2+}$. This was done by heating the pressed ferrite at $1050^{\circ} \mathrm{C}$ (after burning the binder) in the presence of a calculated quantity of reduced iron powder in a closed system. The iron takes up the oxygen given out by the thermal dissociation of the oxidized ferrite and ultimately reduces all the $\mathrm{Mn}^{3+}$ to $\mathrm{Mn}^{2+}$. Subsequently the ferrite was sintered at $1150^{\circ} \mathrm{C}$

† NCL Communication No. 3059

* To whom all communications to be addressed. 
in a static atmosphere of pure nitrogen and in the presence of pellets of active ferric oxide. The $\mathrm{Fe}_{2} \mathrm{O}_{3}$ generates the partial pressure of oxygen necessary to produce the optimum amount of $\mathrm{Fe}^{2+}$ required in the ferrite (Winkler 1971; Ohta 1963).

Volatilizable binders as a rule do not possess the binding strength of PVA which because of its hydrogen bonding - can press with advantage complicated shapes such as pot-cores. But if PVA is employed for pressing, and subsequently burnt out with a simultaneous inevitable oxidation of $\mathrm{Mn}^{2+}$, all the effort spent so far to keep $\mathrm{Mn}^{2+}$ as such is in principle wasted. Besides, there arises a serious problem - that of cracks in the sintered pieces - whenever oxidation and reduction are carried out on the pressed ferrites. This was indeed noticed on performing a large number of sintering runs of the type described in paper 2 and briefly sketched in the last paragraph. It was therefore necessary to study in greater detail the sintering in a static atmosphere, of manganouszinc ferrites pressed with PVA.

\section{Experimental}

\subsection{Synthesis from different manganese oxides}

2.1a Synthesis from $\mathrm{MnO}: \mathrm{MnO}$ was stabilized (Deshpande et al 1978; Deshpande and Murthy 1981) by solid solution with the required amount of $\mathrm{ZnO}$ and then reacted at $950^{\circ} \mathrm{C}$ with the ferric oxide in a closed inert atmosphere in order to yield the ferrite powder (paper 2). The $x$-ray powder pattern even at this stage revealed a single phase of pure manganous-zinc ferrite. The powder was pressed with a volatilizable binder such as camphor into toroids $2.2 \mathrm{~cm}$ OD, $1.2 \mathrm{~cm}$ ID and $0.7 \mathrm{~cm}$ thickness. The camphor was then distilled off at $100^{\circ} \mathrm{C}$ in a current of oxygen-free nitrogen. The pressed pieces were then sintered at $1250^{\circ} \mathrm{C}$ for $8 \mathrm{hr}$ in a closed static system filled initially with pure nitrogen and in the presence of double the weight of active ferric oxide pellets prepared as given in paper 2 . The sintered pieces had no cracks and the magnetic measurements on toroids obtained from twenty five such runs are summarized in table 1 . The $x$-ray powder pattern matched fully with that expected for manganous-zinc ferrites. The electron micrograph of this material is given in figure 1.

2.1b Synthesis from a higher oxide of manganese: Active precipitated $\mathrm{MnO}_{2}$ was thoroughly mixed with the required amounts of active $\mathrm{ZnO}$ and $\mathrm{Fe}_{2} \mathrm{O}_{3}$ (prepared as given in paper 2), pressed into pellets and heated at $950^{\circ} \mathrm{C}$ for $8 \mathrm{hr}$ in a closed system filled with pure nitrogen and in the presence of a calculated quantity of reduced iron (kept out of physical contact with the reaction mixture) in order to reduce all the manganese to $\mathrm{Mn}^{2+}$. The $x$-ray powder pattern of this calcined material showed however not only the lines of manganous-zinc ferrite but also additional lines at $d=1.54$ and $2.19 \AA$ A volatilizable binder was again employed to press this material and the sintering of toroids was done exactly as in $\$ 2.1 \mathrm{a}$. Again there were no cracks even in the course of a large number of runs and the results from 25 such runs are summarized in table 2. The x-ray powder pattern of the sintered material however gave an extra peak at $d=2.028 \mathrm{~A}$ besides the lines of manganous-zinc ferrite. The electron micrograph of this material is shown in figure 2. A simple DTA curve in air for the mixture of higher oxide of manganese, $\mathrm{ZnO}$ and $\mathrm{Fe}_{2} \mathrm{O}_{3}$ used in the preparation of this material is given in figure 3. An x-ray powder pattern of the material got by a simple air-firing of the above oxide mixture at $950^{\circ} \mathrm{C}$ for $8 \mathrm{hr}$ showed intense lines of $\mathrm{ZnFe}_{2} \mathrm{O}_{4}, \mathrm{ZnMn}_{2} \mathrm{O}_{4}$ and also some free $\mathrm{Fe}_{2} \mathrm{O}_{3}$. 


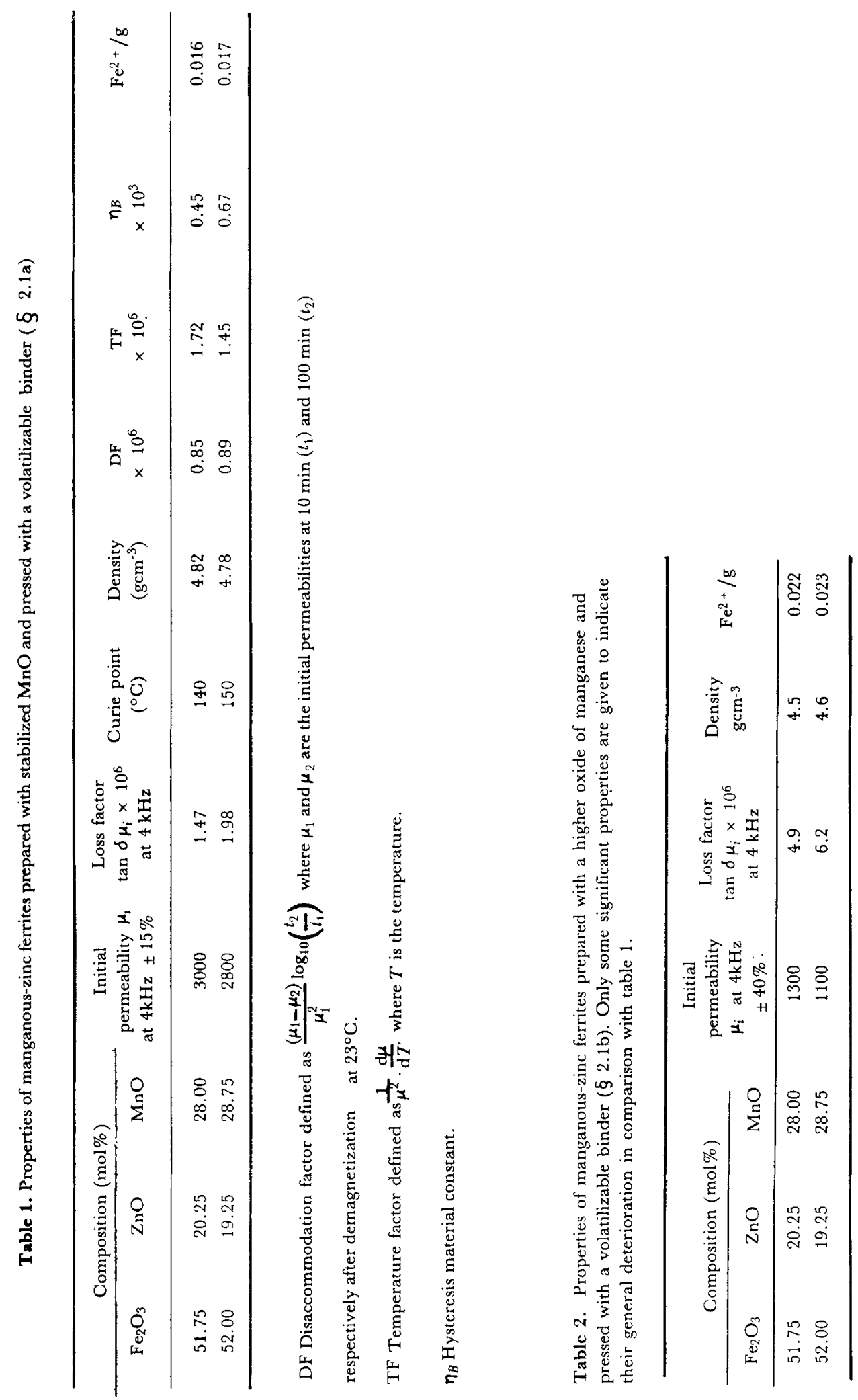




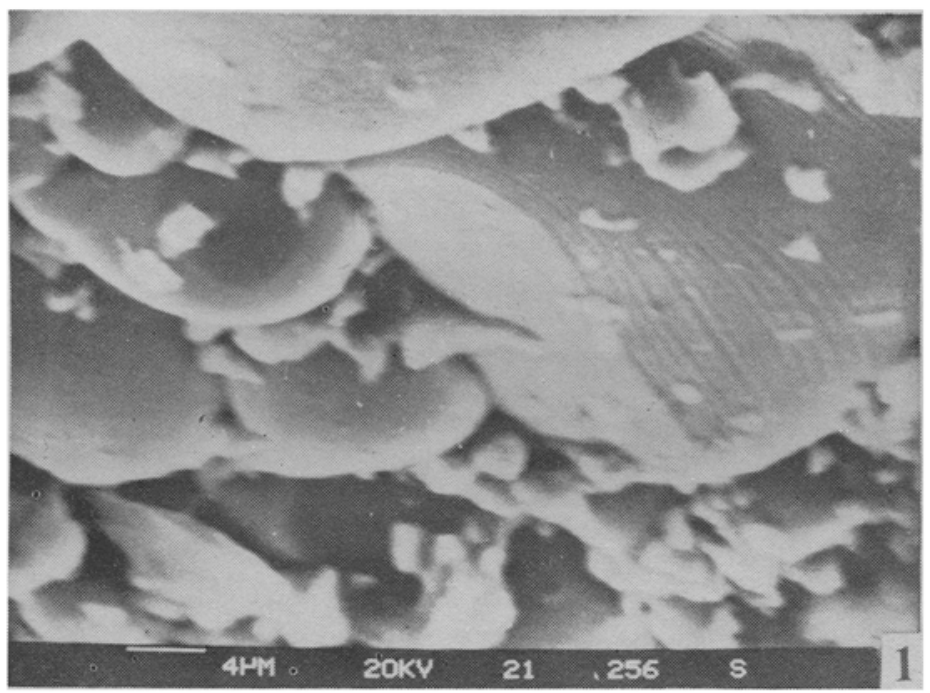

Figure 1. Electron micrograph of manganous-zinc ferrite prepared out of stabilized $\mathrm{MnO}$, pressed with volatilizable binder; binder distilled off and toroids sintered at $1250^{\circ} \mathrm{C}$ in static $\mathrm{N}_{2}$ atmosphere with $\mathrm{Fe}_{2} \mathrm{O}_{3}$ pellets $(\S 2.1 \mathrm{a})$.

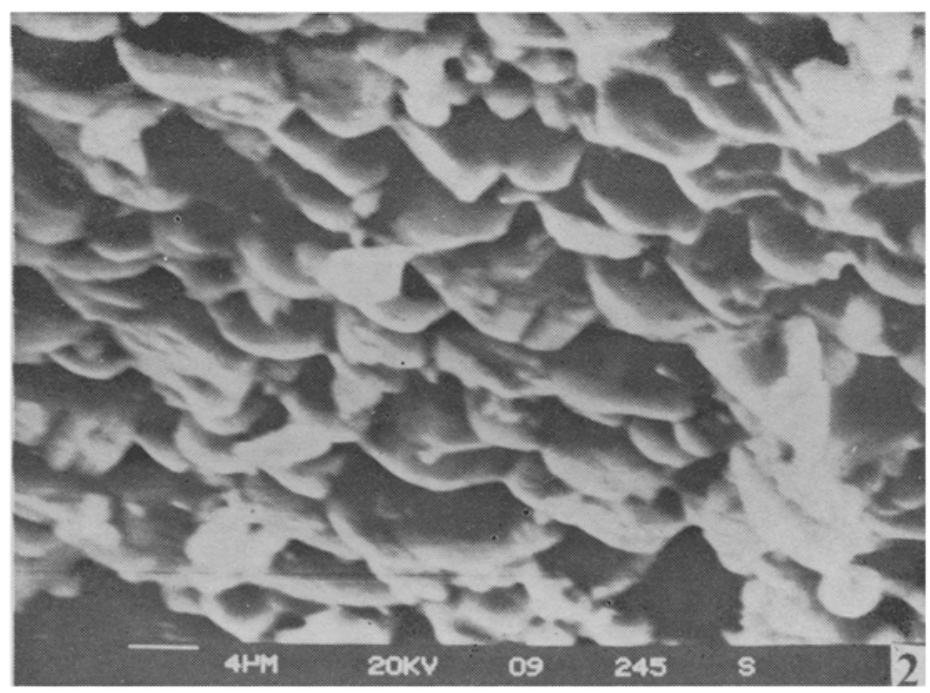

Figure 2. Same legend as for figure 1 except that the ferrite was made out of a higher oxide of manganese which was subsequently reduced $(\S 2.1 \mathrm{~b})$.

\subsection{Use of PVA as binder}

2.2a Burning the binder in air: The ferrite powder was prepared from stabilized $\mathrm{MnO}$ as described in $\S 2.1 \mathrm{a}$, and pressed into toroids with $2 \%$ PVA. It was found, on slow heating of these toroids in air, that the minimum temperature needed for the complete oxidation of the PVA embedded in the ferrite was in a narrow temperature range of $375^{\circ}-400^{\circ} \mathrm{C}$, at which some oxidation of the $\mathrm{Mn}^{2+}$ in the ferrite was inevitable. The 


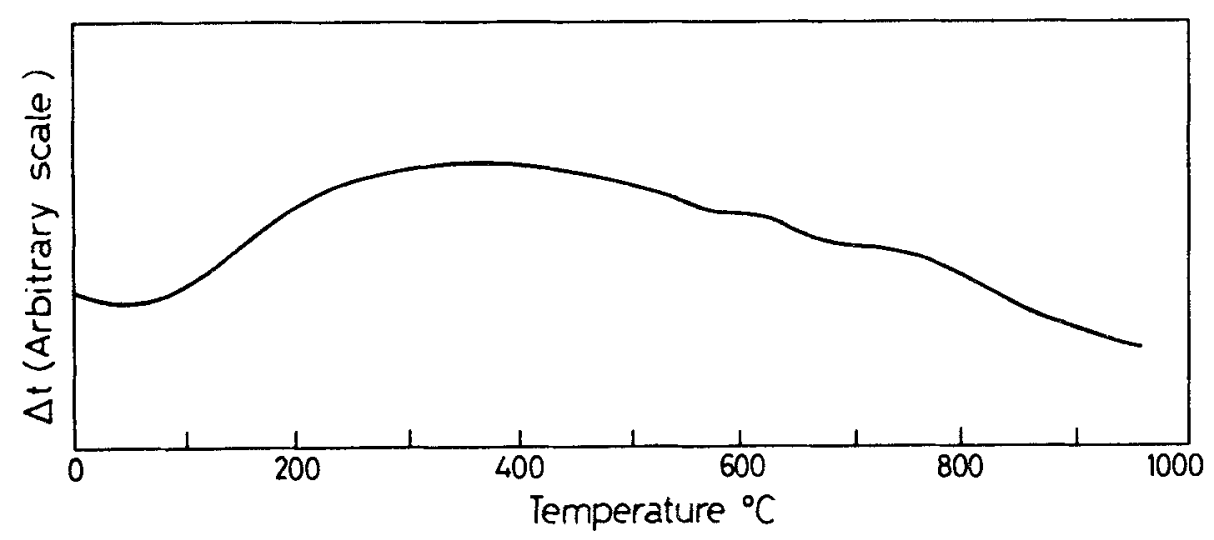

Figure 3. DTA in air of a mixture of higher oxide of manganese $+\mathrm{ZnO}+\mathrm{Fe}_{2} \mathrm{O}_{3}$.

minimum temperature needed for complete combustion of pure PVA (not embedded in the ferrite) was however found to be $\sim 800^{\circ} \mathrm{C}$.

A dozen ferrite toroids pressed with PVA were then heated in air at $400^{\circ} \mathrm{C}$ for $2 \mathrm{hr}$ until the binder was totally burnt out. One of the toroids was broken and the extent of oxidation of $\mathrm{Mn}^{2+}$ was estimated chemically. The partially-oxidized ferrite toroids were reduced in the presence of iron powder in a closed inert atmosphere as mentioned earlier. The toroids were then sintered in a closed system filled with pure nitrogen and in the presence of active ferric oxide pellets (prepared as given in paper 2).

The results from 25 such runs are summarised in table 3 . In each run at least half the number of toroids developed mesh-like cracks.

2.2b $\mathrm{Fe}_{2} \mathrm{O}_{3}$ pellets for burning the binder : Manganous-zinc ferrite prepared from stabilized $\mathrm{MnO}(\xi 2.1 \mathrm{a})$ was pressed into toroids with $2 \%$ PVA A dozen of these toroids was directly sintered at $1250^{\circ}$ for $8 \mathrm{hr}$ in the presence of double the weight of active ferric oxide pellets in a closed unit initially filled with pure nitrogen (unit shown in figure 2 of paper 2). It was found that the binder had completely burnt off and none of the toroids had any cracks even in the course of 25 such runs. The results are given in table 4 . The reproducibility was very good.

2.2c Sintering in air: The preparation of the ferrite and sintering were done exactly as described in $\zeta 2.2 \mathrm{~b}$ except that the closed sintering unit contained air and not nitrogen to start with. The results were as in $\oint 2.2 \mathrm{~b}$ and there were no cracks. The electron micrograph of this material is shown in figure 4 . The $x$-ray powder pattern of the sintered material showed only the characteristic lines of manganous-zinc ferrites.

2.2d Sintering in oxygen: The entire procedure was exactly as in $\S 2.2 c$ except that oxygen was filled in the closed sintering unit before starting the sintering run. The results are given in table 5 . There were no cracks. Figure 5 gives the electron micrograph of this material.

\section{Discussion}

The calcined ferrite prepared out of a higher oxide of manganese $(\S 2.1 \mathrm{~b})$ as the source of manganese gives clear extra peaks in its $x$-ray diffraction pattern as compared with 

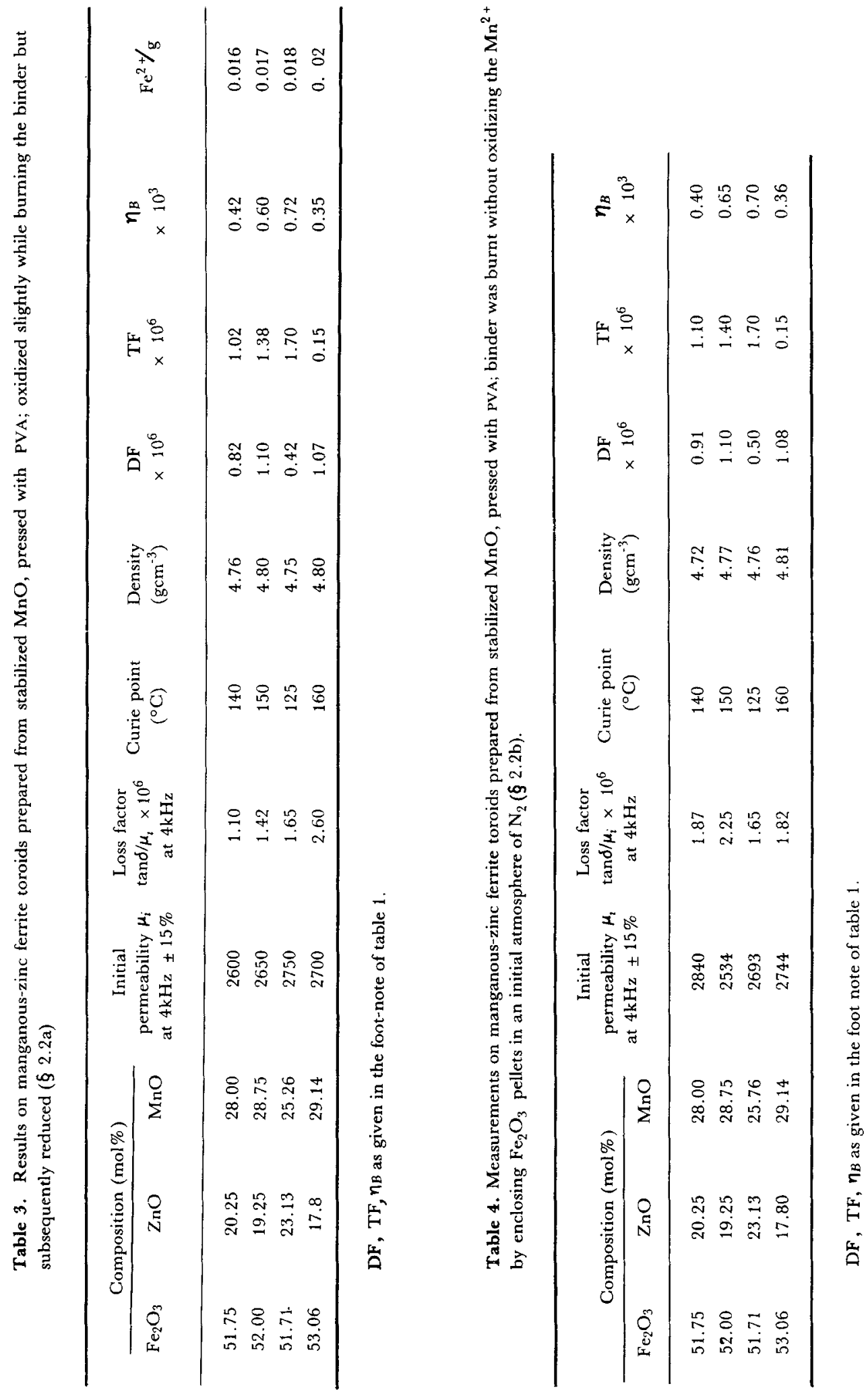


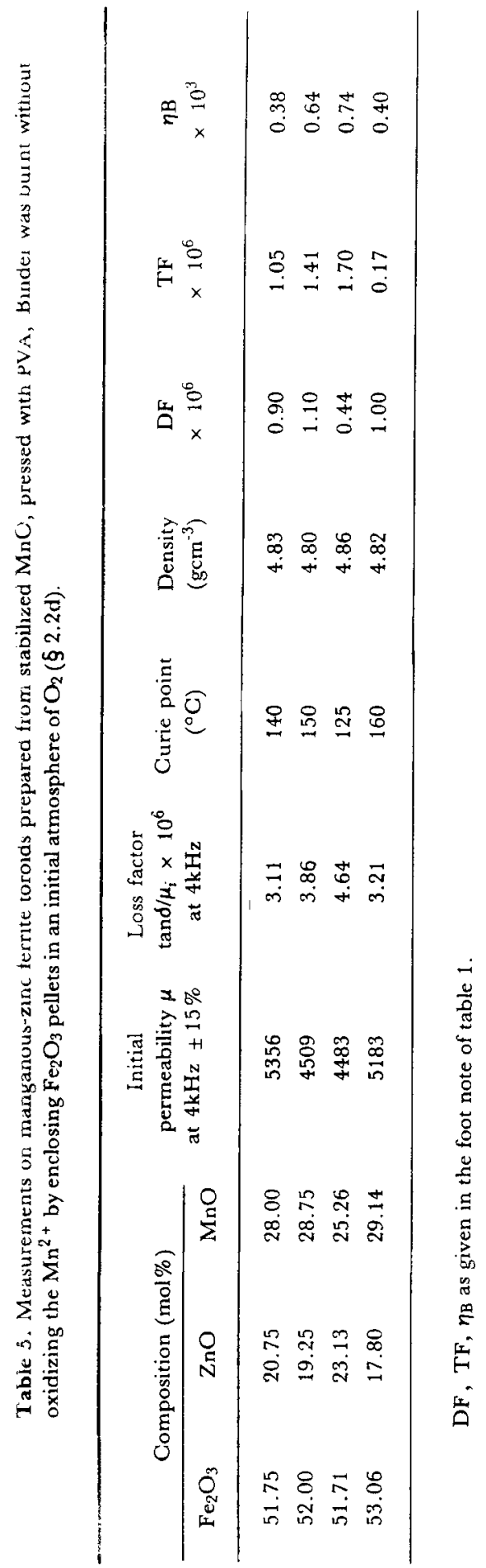




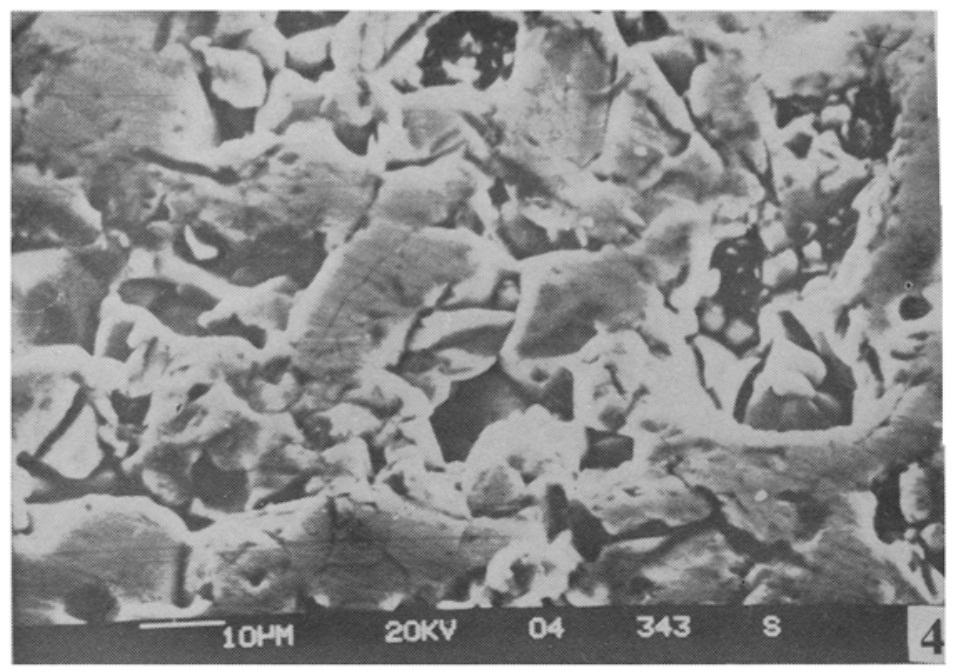

Figure 4. Electron micrograph of manganous-zinc ferrite prepared out of stabilized $\mathrm{MnO}$, pressed with PVA sintered directly in a closed static atmosphere of air in the presence of $\mathrm{Fe}_{2} \mathrm{O}_{3}$ pellets. $(\oint 2.2 \mathrm{c})$

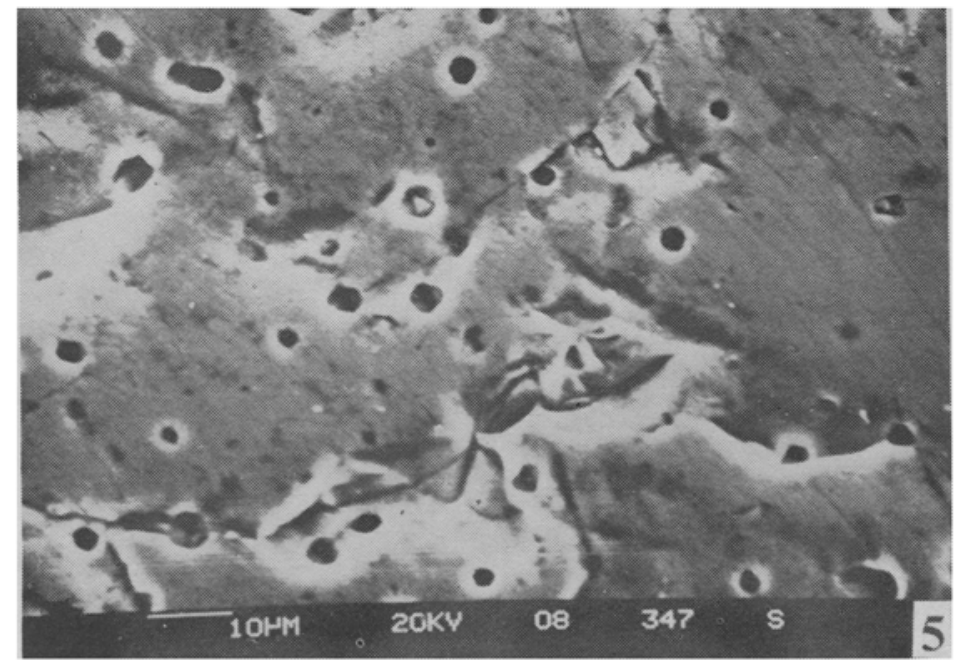

Figure 5. Same legend as for figure 4 except that air was replaced with oxygen $(\oint 2.2 \mathrm{~d})$.

that made out of stabilized $\mathrm{MnO}(\S 2.1 \mathrm{a})$. The peak at $d=2.19 \AA$ is the most intense line and another line at $d=1.54 \AA$ is also a fairly strong line of $\mathrm{MnO}$. MnO also gives a fairly strong line at $d=2.57 \AA$; but this would have merged with the strongest line of the ferrite at $d=2.54 \AA$. The $\mathrm{x}$-ray powder patterns of the calcined ferrites clearly show therefore that $\mathrm{MnO}$ can straightaway enter into the spinel formation on reacting with $\mathrm{ZnO}$ and $\mathrm{Fe}_{2} \mathrm{O}_{3}$; while there are clear difficulties in starting with a higher oxide of manganese even though the latter may be reduced to $\mathrm{MnO}$ afterwards. The DTA curve (figure 3 ) shows that there is appreciable reaction of the higher oxide of manganese 
with $\mathrm{ZnO}$ and $\mathrm{Fe}_{2} \mathrm{O}_{3}$ even at $300^{\circ} \mathrm{C}$. Any reduction of the higher oxide of manganese to $\mathrm{MnO}$ can take place at a much higher temperature than $300^{\circ} \mathrm{C}$ - the temperature at which a good deal of unwanted $\mathrm{ZnMn}_{2} \mathrm{O}_{4}$ has been formed as shown by the $\mathrm{x}$-ray patterns $(\S 2.1 \mathrm{~b})$. The valencies may get corrected chemically; but once an ion with a wrong initial valency gets into a certain lattice site preferred by the starting valency, the rigidity of the crystal lattice does not permit easy migration of the same ion, after reduction, to its new preferred lattice site. It is significant that even the sintered ferrite $\left(\oint_{2} .1 \mathrm{~b}\right)$ still gives an extra peak at $d=2.028 \AA$ which is actually the strongest line of $\mathrm{Mn}_{3} \mathrm{O}_{4}$. It is not unlikely that this material also contains some other unwanted compounds too small in quantity to be detected by $x$-ray diffraction. Starting with a higher oxide of manganese for preparing manganese zinc ferrites therefore has a distinct disadvantage; the solid state reaction precedes the correction of the valency of manganese; and since the site preference of a metal ion in the solid state compound is determined by the valency of the ion at the time of reaction, some unwanted compounds as well as some unwanted spinels such as $\mathrm{Mn}_{3} \mathrm{O}_{4}$ or even the ferrite with undesirable cation distributions in its lattice sites cannot be avoided in such a preparative process. This explains the less satisfactory and less reproducible magnetic properties of table 2 as compared to table 1 corresponding to the ferrite prepared out of stabilized $\mathrm{MnO}$. It is also significant that the electron micrograph of the ferrite prepared as per $\oint 2.1 \mathrm{~b}$ (out of a higher oxide of manganese) contains smaller grains (figure 2) than those (figure 1) in the ferrite prepared as per $\oint 2.1$ a (out of stabilized $\mathrm{MnO}$ ). This is obviously because the stabilized $\mathrm{MnO}$ has already given the ferrite grains even at the stage of calcination (as shown by the $x$-ray powder pattern of this material); and these grains can grow without any hindrance while sintering. The powder pattern of the calcined ferrite made out of a higher oxide of manganese ( $(2.1 \mathrm{~b})$ shows on the other hand some lines of $\mathrm{MnO}$ also. That means the spinel formation in this case is incomplete at the stage of calcination: some further structural changes have taken place subsequently while sintering. A proper grain growth under such conditions perhaps needs a higher temperature than has been used here $-1250^{\circ} \mathrm{C}$. In fact the ferrite prepared out of a higher oxide of $\mathrm{Mn}$ - usual commercial method - is normally sintered above $1300^{\circ} \mathrm{C}$. Such high temperatures are however not desirable for this ferrite which contains the thermolabile $\mathrm{ZnO}$. There would be still another factor contributing to the poorer grain growth as shown in figure 2. The reduction of the valency of manganese during the sintering run means the simultaneous evolution of oxygen from the body of the ferrite; and other things being equal this causes a breaking up of the ferrite grains.

The main object of the experimental work reported in $\S 2.1$ (and being discussed in the present section) was to compare the performance of $\mathrm{MnO}$ as raw material for the preparation of manganous-zinc ferrite with that of a higher oxide of manganese. The use of a non-volatilizable binder at this stage would not have helped in isolating the problem of the starting valency of manganese; volatilizable binders have therefore been employed at this stage. Another point to be mentioned here is that the method of reduction of the valency of manganese at the calcination stage used in this work is different from the conventional method of passing a current of inert gas over the heated reaction mixture (Natansohn and Baird (1969); Strivens and Chol (1971)). This latter method has been deliberately avoided in this work since it entails a greater loss of the volatile $\mathrm{ZnO}$. A last point to be noticed in this section is that the problem of cracks has not appeared at this stage though the initial valency of manganese has been reduced subsequently during the process. The reduction has taken place at the stage of calcination 
and not on the pressed pieces. So long as there is no oxidation or reduction of the material after pressing, there are no volume changes (Economos 1955) and no stresses in the pressed material being sintered.

\subsection{Use of PVA to press $M n-Z n$ ferrite}

The PVA embedded in the ferrite gets completely oxidized below $400^{\circ} \mathrm{C}$ - while in the absence of the ferrite pure PVA takes $\sim 800^{\circ} \mathrm{C}$ to burn off. This is obviously due to the catalytic action of the ferrite. It has been shown ( $\$ 2.2 \mathrm{a})$ that a good deal of organic matter belonging to the binder disappears in a narrow temperature range. Apparently the catalytic oxidation of organic matter in this narrow range of temperature must be very fast; perhaps so fast that the escaping gases cleave through the ferrite matrix thereby initiating cracks which may develop further while sintering. Even a very slow programming of temperature while burning the binder helped little to avoid these cracks which however decreased in number and in extent by controlling the oxygen available for the combustion (Deshpande 1982). Apparently this is not the only reason for the cracks in the sintered pieces; cracks persisted as long as the binder could not be oxidized without a simultaneous oxidation of the $\mathrm{Mn}^{2+}$. The latter would require a partial pressure of oxygen sufficient to oxidize the organic matter but not the $\mathrm{Mn}^{2+}$ in the ferrite; and the thermal dissociation of active ferric oxide pellets $(\xi 2.2 \mathrm{~b})$ provides such an optimum oxygen partial pressure. The dissociation pressure of ferric oxide also controls the ferrous content in the ferrite (as described in paper 2). This ferrous content needs to be controlled optimally in order to minimize the magnetocrystalline anisotropy and magnetostriction so that the permeability increases (Winkler 1971; Ohta 1963).

It has been observed (Economos 1955) previously that any process of oxidation or reduction on the pressed ferrite pieces is liable to produce cracks due to the consequent volume changes and stresses. Even in the course of a hundred sintering runs of the type described in $\xi 2.2 \mathrm{~b}$, no cracks were observed and the results were highly reproducible. The presence of air to start with, instead of nitrogen, in the closed sintering unit made no difference to the outcome of the sintering runs. The limited air enclosed in the sintering unit was far from sufficient to burn the binder and the oxygen needed for burning off the binder had to come, by and large, from $\mathrm{Fe}_{2} \mathrm{O}_{3}$ even in this case. The air initially present did no harm since a competition between the organic binder and $\mathrm{Mn}^{2+}$ in the ferrite for the limited oxygen filling the unit went as expected in favour of the organic binder. It was even found possible to carry out the above sintering run $(\S 2.2 \mathrm{~d})$ with oxygen filling the closed sintering unit provided this oxygen was too insufficient to burn the PVA present. The corresponding improved results given in table 5 and better grain growth shown in figure 5 can perhaps be explained on the basis of increased cation vacancies which in turn enhance the mobility of cations. At least part of the $\mathrm{Fe}_{2} \mathrm{O}_{3}$ present over and above $50 \mathrm{~mol} \%$ in the ferrite changes to the $\gamma$ variety which may be in equilibrium with $\mathrm{Fe}_{3} \mathrm{O}_{4}$ (Slick 1980).

$$
\begin{aligned}
& 4 \mathrm{Fe}_{2}^{3+} \mathrm{O}_{3}(\mathrm{rh}) \rightarrow 3 \mathrm{Fe}_{8 / 3}^{3+} \square_{1 / 3} \mathrm{O}_{4}(\mathrm{sp}) \\
& 3 \mathrm{Fe}_{8 / 3}^{3+} \square_{1 / 3} \mathrm{O}_{4} \rightleftharpoons 8 / 3 \mathrm{Fe}^{2+} \mathrm{Fe}_{2}^{3+} \mathrm{O}_{4}+2 / 3 \mathrm{O}_{2}
\end{aligned}
$$

The presence of oxygen in the atmosphere shifts the second equilibrium backwards and causes cation vacancies which are favourable to grain growth. 


\section{Conclusion}

Manganous-zinc ferrites are best prepared directly from stabilized $\mathrm{MnO}$. The ferrites pressed with PVA are best sintered directly in the static atmosphere of a closed system and in the presence of a sufficient number of active ferric oxide pellets. These ferric oxide pellets need not be very pure since they do not come in contact with the ferrite. They may be prepared by dissolving scrap iron in $30 \%$ nitric acid and processing the ferric nitrate as given in paper 2 . The used ferric oxide can be reprocessed by dissolving in hydrochloric acid and precipitating the ferric hydroxide with an alkali. The static atmosphere in the closed sintering unit can be air itself or even oxygen so long as the oxygen is too insufficient to burn the binder. This method of sintering completely eliminates cracks besides giving highly satisfactory and reproducible properties.

\section{Acknowledgement}

The authors are grateful to the Prototype Production Unit of the Armament Research and Development Laboratory, for the excellent dies which they prepared and for their help in pressing the ferrites. They are grateful to Mrs J J Shrotri for general help in the work, to Shri J S Gujral for scanning the $\mathrm{x}$-ray diffractograms and to Mrs A Mitra for taking the electron micrographs. The authors are also grateful to Dr A P B Sinha for encouraging this work.

\section{References}

Deshpande C E 1982 Unstable oxides $\mathrm{MnO}$ and $\mathrm{FeO}$; their stabilization and use in some ferrites, Ph.D. Thesis, University of Poona.

Deshpande C E and Murthy M N S 1981 Bull. Mater. Sci. 3261

Deshpande C E, Pant L M and Murthy M N S 1978 Indian J. Chem. A16 251

Economos G $1955 \mathrm{~J} . \mathrm{Am}$. Ceram. Soc. 38241

Murthy M N S, Deshpande C E and Shrotri J J 1978 Proc. Indian Acad. Sci. A 8749

Murthy M N S, Deshpande C E, Bakare P P and Shrotri J J 1979 Bull. Chem: Soc. Jpn. 52571

Natansohn S and Baird D H 1969 J.Am.Ceram. Soc. 52129

Ohta K 1963 J. Phys. Soc.Jpn. 18685

Slick P I 1980 Ferromagnetic materials (ed) E P Wohlfarth (New York: North-Holland Publishing Co) Vol. II p.202

Strivens M A and Chol G 1971 Ferrites Proc. Int. Conf. Jpn 1970 (eds) Y Hoshiro, S Iida and M Sugimoto (Tokyo: University Press) p. 239

Winkler G 1971 Magnetic properties of materials (ed) J Smit (New York: McGraw Hill) p.52 